Background Estimating the distribution of new HIV infections according to identifiable characteristics is a priority for programmatic planning in HIV prevention. We propose a mathematical modelling approach that uses robust data sources to estimate the distribution of new infections acquired in the generalised epidemics of sub-Saharan Africa and validate it against cohort data. Methods We developed a predictive model that represents the population according to factors powerfully associated with risk: gender, marital status, geographic location, key risk behaviours (sex-work, injecting drug-use, male-to-male sex), sero-discordancy within couples, circumcision and ART status. Incidence inference methods are applied to estimate the short-term distribution of new infections by group. The model is applied within a Bayesian framework whereby regional demographic and epidemiological prior information is updated, where possible, with local data. We validated and trained the model against cohort data from Manicaland (Zimbabwe), Kisesa (Tanzania) and Rakai (Uganda). Building on the results from the acquisition model we infer likely sources of transmission. The model was applied to six countries in the region to investigate potential differences in incidence patterns.

Results Without training using the site-specific data, the model was able to predict the pattern of new infections with reasonable accuracy: 95\% credible intervals were substantially overlapping and the rank ordering of groups with new infections was consistent. With training using group-specific data on new infections, the accuracy of predictions for subsequent rounds of data improved further and credible intervals narrowed. When applied to the six countries in the region the model showed variation in the distribution of infections between and within countries consistent with the data on prevalence.

Conclusions It is possible to accurately predict, the distribution of new HIV infections acquired using data routinely available in many countries in the Sub-Saharan African region. This validated tool can complement additional analyses on resource allocation and data collection priorities.

Declaration of conflicts of interest All authors declare having no conflicts of interest.

\section{P16.12 OPTIMISATION HIV INVESTMENT IN SWAZILAND: MODELLING HIGH-IMPACT INTERVENTIONS}

${ }^{1}$ SL Kelly*, ${ }^{1} \mathrm{AJ}$ Shattock, ${ }^{1,2} \mathrm{CC}$ Kerr, ${ }^{1} \mathrm{DP}$ Wilson, ${ }^{3} \mathrm{~T}$ Gama, ${ }^{3} \mathrm{~N}$ Mathabela, ${ }^{4} \mathrm{~N}$ Ceesay, ${ }^{5} \mathrm{M}$ Gorgens. ${ }^{1}$ The Kirby Institute; ${ }^{2}$ University of Sydney; ${ }^{3}$ NERCHA; ${ }^{4}$ UNAIDS; ${ }^{5}$ World Bank

10.1136/sextrans-2015-052270.559

Introduction Swaziland has the highest global HIV prevalence with an estimated $27 \%$ of people aged 15-49 living with HIV. To address this issue, the Government aimed to optimise HIV investment by assessing the impact of key interventions.

Methods We used Optima to assess the impact of moderate- and high-level scale-up of five interventions: scaling-up antiretroviral therapy (ART) coverage to $65 \%$ (at CD4 count $<500$ cells $/ \mathrm{mm}^{3}$ ) by 2020 and $75 \%$ by 2030 (moderate scale-up) or $85 \%$ by 2020 and $90 \%$ by 2030 (high-level scale-up), voluntary medical male circumcision (VMMC) to $55 \%$ by 2018 (moderate) or $45 \%$ by 2015 and $70 \%$ by 2018 (high-level) for males aged $10-49$, prevention of mother-to-child transmission to $90 \%$ (moderate) or $95 \%$ (high-level) by 2018, tuberculosis/HIV co-treatment to $75 \%$ by $2015,85 \%$ by 2018 , and $90 \%$ by 2030 (moderate) or similar targets for high-level scale-up except with $95 \%$ coverage by 2030 , and implementation of conditional cash transfers
(CCT) to 60\% (moderate) or 95\% (high-level) by 2018 for women aged 15-24. Data were provided by the Swaziland Government for general and key populations disaggregated by age and sex for 2000-2013.

Results By 2030, compared to current coverage, it is possible to reduce new infections by $27 \%$ and AIDS-related deaths by $12 \%$ (moderate scale-up) or by $49 \%$ and $24 \%$, respectively, with highlevel scale-up. The highest impact interventions are ART, VMMC, and CCT.

The discounted cumulative additional program cost of these combined interventions was US\$74 million with an incremental cost-effectiveness ratio of US $\$ 2,700$ per infection averted (for moderate scale-up) and US\$309 million with an incremental cost-effectiveness ratio of US\$6,300 per infection averted (for high level scale-up).

Conclusion Rapid scale-up of ART and VMMC, as well as implementation of CCT for women aged 15-24 showed the greatest impact on reducing new HIV infections and AIDS-related deaths in Swaziland.

Disclosure of interest statement No conflicts of interest.

\section{P16.13 EPIDEMIOLOGY OF HIV PREVALENCE AMONG URBAN WOMEN IN TANZANIA}

RK Singh* . International Institute for Population Sciences, Mumbai, India

\subsection{6/sextrans-2015-052270.560}

Introduction Tanzania is the country that was the hardest hit by the HIV epidemic in Sub-Saharan Africa. The present study was carried out to examine the risk factors of HIV infection among women who lived in an urban area in Tanzania.

Methods The Tanzania HIV/AIDS and Malaria Indicator Survey (2011-12) data has been used. The sample size for urban and rural women who were tested for HIV and ever had sex was 2227 and 6210 respectively. Bivariate and multivariate analyses like logistic regression analyses were used.

Results The present study found that rural women were significantly less likely to be HIV-infected compared to urban women $(\mathrm{OR}=0.612, \mathrm{p}<0.00)$. About $10 \%$ urban women were HIVinfected whereas $5.8 \%$ women in rural areas were HIV positive. Those women who had more than five sex partners were significantly four times more likely to be HIV-infected compared to women had one sex partner (OR $=4.49, \mathrm{p}<0.00)$.

Conclusion The present study results suggested that less-educated women, women belong to poor or poorer quintile, women spent night outside, had more sex partners were significantly more likely to had HIV infection among urban women compared to rural women. There is an urgent need for a short and effective program to control the HIV epidemic in urban areas of Tanzania especially for less-educated urban women.

Disclosure of interest Nothing to Disclose.

\section{P16.14 GEOGRAPHY OF AIDS IN THE STATE OF CEARÁ, BRAZIL}

${ }^{1} \mathrm{NL}$ Pedrosa, ${ }^{1} \mathrm{HO}$ Alexandre ${ }^{*},{ }^{1,2} \mathrm{ICV}$ Lima, ${ }^{1} \mathrm{SS}$ Paiva, 1,2VF Santos, ${ }^{1,2} \mathrm{MTG}$ Galvão. ${ }^{1}$ Federal University of Ceará; ${ }^{2}$ Federal University of Pernambuco

\subsection{6/sextrans-2015-052270.561}

Introduction The geographical distribution of AIDS subsidises the understanding of the relationship of the disease with socioeconomic and cultural characteristics, enabling local planning of 